\title{
Accurate Simulations of Lipid Monolayers Require a Water Model With Correct Surface Tension
}

\author{
Carmelo Tempra, ${ }^{\dagger}$ O.H. Samuli Ollila, ${ }^{\ddagger}$ and Matti Javanainen ${ }^{*,+, \dagger}$ \\ $\dagger$ Institute of Organic Chemistry and Biochemistry, Czech Academy of Sciences, Flemingovo \\ nám. 542/2, 16000 Prague 6, Czech Republic \\ $\ddagger$ Institute of Biotechnology, University of Helsinki, 00014 Helsinki, Finland \\ E-mail: matti.javanainen@gmail.com
}

\begin{abstract}
Lipid monolayers provide our lungs and eyes their functionality, and serve as proxy systems in biomembrane research. Therefore, lipid monolayers have been studied intensively also using molecular dynamics simulations, which are able to probe their lateral structure and interactions with, e.g., pharmaceuticals or nanoparticles. However, such simulations have struggled in describing the forces at the air-water interface. Particularly the surface tension of water and long-range van der Waals interactions have been considered critical, but their importance in monolayer simulations has been evaluated only separately. Here we combine the recent C36/LJ-PME lipid force field that includes long-range van der Waals forces with water models that reproduce experimental surface tensions to elucidate the importance of these contributions in monolayer simulations. Our results suggest that a water model with correct surface tension is necessary to reproduce experimental surface pressure-area isotherms and monolayer phase behavior, while standard cutoff-based CHARMM36 lipid model with the 4-point OPC
\end{abstract}


water model still provides the best agreement with experiments. Our results emphasize the importance of using high quality water models in applications and parameter development in molecular dynamics simulations of biomolecules.

\section{Introduction}

Monolayers of amphiphilic lipids serve as a proxy for lipid membranes in studies of membrane structure and membrane-protein interactions, ${ }^{1,2}$ since they are significantly more straightforward to study with wide range of spectroscopic and microscopic methods than lipid bilayers. ${ }^{3,4}$ Moreover, lipid monolayers form functionally essential structures that line the alveoli in the lungs and cover the surfaces of the eyes. ${ }^{5}$ In the lungs, a pulmonary surfactant (PS) monolayer covers the alveolar liquid, preventing the collapse of the alveoli during exhalation. In the eyes, a tear film lipid layer (TFLL) consists of a monolayer that separates the tear fluid from the non-polar wax layer of the TFLL, thus helping the latter spread rapidly between eye blinks. Both PS and TFLL are compositionally complex, likely to optimize their mechanical behavior under dynamic conditions. Notably, this behavior depends on the subtle balance of forces at the liquid-air interface.

A Langmuir trough enables the measurement of lipid monolayer surface tension as a function of its area, thereby providing insighs into the behavior of PS and TFLL. Moreover, monitoring the changes in the resulting surface pressure-area isotherms upon the addition of biomolecules - such as proteins and drugs - into the aqueous subphase can be used to understand their binding to membranes. Above the main transition temperature $\left(T_{\mathrm{m}}\right)$ of the phospholipid, the monolayer remains in the fluid-like liquid expanded $\left(\mathrm{L}_{\mathrm{e}}\right)$ phase over a large range of areas. Below the $T_{\mathrm{m}}$ value, $\mathrm{L}_{\mathrm{e}}$ phase transforms upon compression to a gel-like liquid condensed $\left(\mathrm{L}_{\mathrm{c}}\right)$ phase through a coexistence plateau. ${ }^{6}$ At very large areas, the pores form in the monolayer and a gas $-\mathrm{L}_{\mathrm{e}}$ coexistence appears at very low surface pressures close to $0 \mathrm{mN} / \mathrm{m}$.

Due to their physiological importance, monolayers modeling PS or TFLL have been sub- 
ject to numerous computational studies which have utilized both coarse-grained and atomistic molecular dynamics (MD) simulation approaches. ${ }^{7,8}$ MD simulations are also used to complement spectroscopic monolayer experiments, for example to understand ion binding to membranes. ${ }^{9}$ However, MD simulations have struggled to correctly capture the interactions at interfaces between polar and non-polar environments that provide PS and TFLL their functionality, ${ }^{10,11}$ yet these interactions need to be properly balanced to reproduce experimental pressure-area isotherms. ${ }^{12-14}$ This discrepancy has been suggested to arise from underestimated water-air surface tension of common water models ${ }^{14,15}$ and the truncation of long-range van der Waals interactions which compromises the description of acyl chainvacuum interface. ${ }^{13,16,17}$

We have recently demonstrated that the CHARMM36 ("C36" from now on) lipid model ${ }^{13}$ combined with the 4-point OPC water model ("OPC4" from now on) ${ }^{18}$ provides nearly quantitative agreement with experimental surface pressure-area isotherms of both singlecomponent ${ }^{15}$ and multi-component ${ }^{19}$ lipid monolayers. This is because OPC4 water model reproduces the surface tension of water with a Lennard-Jones (LJ) cutoff of 1.2-1.4 nm that is consistent with common lipid models such as the $\mathrm{C} 36^{13}$ and Slipids ${ }^{20}$ ones. Thus, the OPC4 water model enables more realistic simulations of lipid monolayers without the need to re-parameterize the entire lipid model. However, this approach still suffers from the issues related the missing attractive long-range van der Waals forces due to the truncation of the LJ potential. On the other hand, these long-range van der Waals interactions are included in the recent version of C36 lipid model, coined C36/LJ-PME, ${ }^{16,17,21}$ through a PME-like algorithm. ${ }^{21-23}$ In this model, the glycerol and ester regions of lipids are modified to avoid over-condensation resulting from the increased attraction. ${ }^{22,24,25}$

Our earlier studies ${ }^{14,15}$ suggest that a water model with correct surface tension is necessary to reproduce experimental surface pressure-area isotherms and phase behaviour of lipid monolayers. Inclusion of long-range LJ interactions increase the surface tension of the used CHARMM-specific TIP3P (TIPS3P) water model, ${ }^{26,27}$ albeit not enough for it to match 
experiment. ${ }^{28}$ The C36/LJ-PME was demonstrated to reproduce the experimental surface tensions at three different areas for a DPPC monolayer, ${ }^{16,17}$ yet its ability to reproduce experimental surface pressure-area isotherms or lipid monolayer phase behaviour has not been evaluated.

Here, we aim to understand whether the ability of the water model to reproduce experimental surface tension, the inclusion of long-range van der Waals interactions, or both are critical for the correct description of lipid monolayers in MD simulations. Our results pave the way toward more realistic simulations of lipid monolayers with applications in wide range of fields from surfactant science to membrane biophysics and pharmacology. The methodological advancement following our results is not limited only to monolayer simulations. Indeed, monolayer surface tensions are used as target parameters in the recently introduced automatic parameterization strategy for C36/LJ-PME, ${ }^{17}$ which is expected to have wide range of applications to in biomolecular simulations of systems with complex compositions.

\section{Methods}

We implemented the C36/LJ-PME model in GROMACS and used it to perform simulations of pure air-water interfaces, lipid bilayers, and lipid monolayers - all with multiple water models. All performed simulations are briefly listed in Table 1. The setup, simulation, and analysis protocols are described in detail in the subsections below (other systems) or in the SI (lipid bilayers). All simulations were performed using GROMACS 2020. ${ }^{29}$ For efficiency and consistency with the the CHARMM implementation, all the LJ-PME simulations performed here with GROMACS used the Lorentz-Berthelot combination rules in the real space and the geometric combination rules in the reciprocal space. ${ }^{21-23}$ 
Table 1: Brief summary of the simulations performed in this work.

\begin{tabular}{|c|c|c|}
\hline System & Temperature & Purpose \\
\hline $\begin{array}{l}\text { Air-water interface } \\
8 \times 3 \times 5 \times 10 \mathrm{~ns}=1.2 \mu \mathrm{s}\end{array}$ & $\begin{array}{l}298,310, \\
\text { and } 323 \mathrm{~K}\end{array}$ & $\begin{array}{l}\text { Evaluate } \gamma_{0} \text { of } 8 \text { different water models with } 4 \\
\text { different LJ cutoffs }(0.8-1.4 \mathrm{~nm}) \text { and LJ-PME }\end{array}$ \\
\hline $\begin{array}{l}\text { POPC bilayers } \\
3 \times 5 \times 300 \mathrm{~ns}=4.5 \mu \mathrm{s}\end{array}$ & $\begin{array}{l}298,303,308 \\
313, \text { and } 318 \mathrm{~K}\end{array}$ & $\begin{array}{l}\text { Validate our C36/LJ-PME implementation } \\
\text { and study its compatibility with } 3 \text { water models }\end{array}$ \\
\hline $\begin{array}{l}\text { DPPC bilayers } \\
4 \times 5 \times 300 \mathrm{~ns}=6.0 \mu \mathrm{s}\end{array}$ & $\begin{array}{c}323,328,333, \\
338, \text { and } 343 \mathrm{~K}\end{array}$ & Also simulated with standard C36 + TIPS3P \\
\hline $\begin{array}{l}\text { POPC monolayers } \\
3 \times 10 \times 200 \mathrm{~ns}=6.0 \mu \mathrm{s}\end{array}$ & $298 \mathrm{~K}$ & $\begin{array}{l}\text { Compare C36/LJ-PME with experimental } \\
\text { isotherms at } 10 \text { areas and with three water models }\end{array}$ \\
\hline $\begin{array}{l}\text { DPPC monolayers } \\
3 \times 14 \times 300 \mathrm{~ns}=12.6 \mu \mathrm{s}\end{array}$ & $298 \mathrm{~K}$ & $\begin{array}{c}\text { Compare C36/LJ-PME with experimental } \\
\text { isotherms at } 14 \text { areas and with } 3 \text { water models }\end{array}$ \\
\hline
\end{tabular}

\subsection{Implementation of C36/LJ-PME Parameters Into GROMACS}

We first implemented the "Linkage" versions of the DPPC and POPC C36/LJ-PME models to GROMACS-compatible formats with TopoGromacs ${ }^{30}$ starting from the CHARMMcompatible files downloaded from https://terpconnect.umd.edu/ jbklauda/ff.html. This version of C36/LJ-PME presents minimal changes from the C36 lipid model, and only the non-bonded parameters of the glycerol and ester groups were optimized, along with changes in the respective dihedral parameters. Thus, for both DPPC and POPC, a total of 17 partial charges, 2 Lennard-Jones parameters, and 33 dihedrals differ from their parametrizations in the standard C36 model. ${ }^{13,16,17}$ The modified GROMACS-compatible topology files are available in the SI.

We validated the parameter conversion by performing identical $300 \mathrm{~ns}$ simulations of DPPC bilayer with OpenMM ${ }^{31}$ using C36/LJ-PME parameters in the original and in the GROMACS-converted formats. These simulations provided essentially identical APL values of $62.5 \pm 0.2 \AA^{2}$ and $62.7 \pm 0.1 \AA^{2}$, respectively. 


\subsection{Surface Tension of Water Models}

The surface tensions of eight commonly employed water models were evaluated at different temperatures and with different LJ treatments by simulating the air-water interface.

We first generated a simulation box with 20052 water molecules and dimensions of $12 \times 12 \times 4 \mathrm{~nm}^{3}$. Next, the shortest box vector was extended to $22 \mathrm{~nm}$ in order to create two interfaces between air (or vacuum) and water. This procedure was repeated for 3-point and 4point water models. Then, we simulated the systems using various 3 -point and 4-point water models; 3-point ${ }^{32}$ (OPC3) and 4-point ${ }^{18}$ Optimal Point Charge (OPC4) models, Simple Point Charge (SPC) ${ }^{33}$ and its Extended variant (SPC/E), ${ }^{34}$ Three-site Transferrable Intermolecular Potential (TIP3P) ${ }^{26}$ and its CHARMM-variant (TIPS3P), ${ }^{27}$ Four-site Transferrable Intermolecular Potential (TIP4P), ${ }^{26}$ and its updated variant from 2005 (TIP4P/05). ${ }^{35}$

The simulations were performed in constant volume and temperature for $10 \mathrm{~ns}$ with varying cutoff values for the Lennard-Jones potential. The simulations used a 2 fs time step. Buffered Verlet lists were used to keep track of atomic neighbours. ${ }^{36}$ Electrostatic interactions were calculated using the smooth Particle Mesh Ewald algorithm. ${ }^{37,38}$ For the LennardJones potential, we used different cutoff values of $0.8,1.0,1.2$, and $1.4 \mathrm{~nm}$. CHARMM force fields use a switch function for the LJ potential, but this would introduce an extra parameter - the distance at which the switching begins - and thus we decided to always shift the potential to zero at the cutoff. We applied dispersion corrections ${ }^{39}$ to energy and pressure, as these correction is used for monolayer simulations with CHARMM. However, the

effect of dispersion corrections on the water-air surface tension is within the error estimate. ${ }^{15}$ We also repeated the simulations using LJ-PME. ${ }^{22,23}$ In all simulations, temperature was controlled by the stochastic velocity rescaling algorithm ${ }^{40}$ with a target temperature of either 298, 310, or $323 \mathrm{~K}$, and a time constant of $1 \mathrm{ps}$. The geometry of the water molecules was constrained by the SETTLE algorithm. ${ }^{41}$

The surface tension values were extracted from pressure components normal $\left(P_{\mathrm{N}}\right)$ and 
lateral $\left(P_{\mathrm{L}}\right)$ to the interface as

$$
\gamma_{0}=\frac{\left(P_{\mathrm{N}}-P_{\mathrm{L}}\right) \times L_{z}}{2} .
$$

Here, $P_{\mathrm{L}}=1 / 2 \times\left(P_{x x}+P_{y y}\right)$, and $P_{x x}=P_{y y}$ due to symmetry, and the length of the simulation box in the direction normal to the interface is $L_{z}$. The surface tension values were extracted with gmx energy, and the standard error was obtained from block averaging performed by gmx analyze. The last $9.9 \mathrm{~ns}$ of the $10 \mathrm{~ns}$-long simulations were used for analysis.

\subsection{Lipid Monolayer Simulations}

Standard setup with two monolayers separated by a slab of water on one side and by a large vacuum space on the other side was used to simulate DPPC and POPC monolayers. The starting structures were taken from our previous work. ${ }^{15}$ The simulations were performed in the canonical ensemble (constant volume, temperature, and particle number) and with periodic boundary conditions in all directions. Monolayers were simulated at different areas per lipid to construct surface pressure-area isotherms that are readily comparable to experiments.

For both DPPC and POPC, simulations were performed with TIPS3P, ${ }^{27}$ the 4 -point OPC, ${ }^{18}$ and TIP $4 / 05^{35}$ water models. These models were chosen, as the last two of them show the best agreement with experimental water-air surface tension values, whereas the first one is the standard water model of the C36/LJ-PME approach. The simulated DPPC monolayers had areas per lipid of 51, 54, 57, 60,63, 66, 69, 72, 75, 78, 86, 94, 102, and $110 \AA^{2}$, and thus cover the $\mathrm{L}_{\mathrm{c}}, \mathrm{L}_{\mathrm{e}}, \mathrm{L}_{\mathrm{c}} / \mathrm{L}_{\mathrm{e}}$, and $\mathrm{L}_{\mathrm{e}}$ /gas regions of the experimental isotherms. The POPC monolayers had areas of 58, 64, 70, 78, 86, 94, 102, 110, 118, and $126 \AA^{2}$, covering the $\mathrm{L}_{\mathrm{e}}$, and $\mathrm{L}_{\mathrm{e}}$ /gas regions. The simulations were either $300 \mathrm{~ns}$ (DPPC) or $200 \mathrm{~ns}$ (POPC) long, and the first $100 \mathrm{~ns}$ were omitted from the analyses, based on the convergence analyses 
from our recent monolayer work. ${ }^{15}$

The equations of motion were integrated with a leap-frog integrator and with a time step of 2 fs. We used buffered Verlet lists ${ }^{36}$ to keep track of atomic neighbours. The smooth $\mathrm{PME}^{37,38}$ and LJ-PME ${ }^{22,23}$ approaches were used to evaluate the long-range electrostatic and van der Waals interactions. The temperatures of the lipid and the solvent were coupled separately to a Nosé-Hoover thermostat ${ }^{42,43}$ with a time constant of 1 ps. P-LINCS ${ }^{44,45}$ was used to constrain bonds involving hydrogen atoms. The geometric combination rules were used for LJ-PME, in line with the CHARMM implementation of C36/LJ-PME. ${ }^{21}$

The surface pressure of the monolayer $\Pi$ at an area per lipid of APL was extracted from the surface tensions of the pure water-air interface $\left(\gamma_{0}\right)$ and the lipid monolayer-coated water-air interface $(\gamma(\mathrm{APL}))$ as

$$
\Pi(\mathrm{APL})=\gamma_{0}-\gamma(\mathrm{APL}) .
$$

The values of $\gamma$ were extracted using gmx energy, and the standard errors were obtained from block averaging performed by gmx analyze. The $\gamma_{0}$ values were taken from the simulations of the pure air-water interface with the corresponding water model. The error of $\Pi$ was estimated as the sum of the standard errors of the corresponding $\gamma_{0}$ and $\gamma$ values.

The phase identity of each lipid was determined by clustering the $10^{\text {th }}$ carbon atoms of the DPPC chains using the DBSCAN algorithm. ${ }^{46} \mathrm{~A}$ chain was considered to be part of the $\mathrm{L}_{\mathrm{c}}$ phase, if it had 6 neighbors within $0.71 \mathrm{~nm}$ in the plane of the monolayer.

\section{Results and Discussion}

First we evaluated our GROMACS implementation of C36/LJ-PME by comparing the area per lipid of DPPC bilayers at different temperatures with the data from the original C36/LJPME publications, ${ }^{16,17}$ our standard C36 simulations, and with experiments (Fig. S1 in the SI). All simulations give consistently slightly lower area per molecule than experiments for 
DPPC bilayer at $333 \mathrm{~K}$. However, at $323 \mathrm{~K}$, C36/LJ-PME simulated with GROMACS goes into a ripple phase and gives significantly lower area per molecule than when simulated with OpenMM. More condensed membranes and higher melting temperatures have been reported also previously from C36 simulations with GROMACS and leap-frog integrator than from OpenMM and Langevin integrator. ${ }^{47,48}$ In Fig. S2 in the SI, we demonstrate that switching to Langevin integrator in GROMACS can melt the ripple phase in DPPC bilayer at $323 \mathrm{~K}$, suggesting that integrators are the source of the difference. For more detailed discussion on the bilayer results, see SI. We conclude that our C36/LJ-PME GROMACS implementation with leap-frog integrator behaves well in temperatures that are not close to phase transition temperatures, similarly to the regular C36 GROMACS implementation.

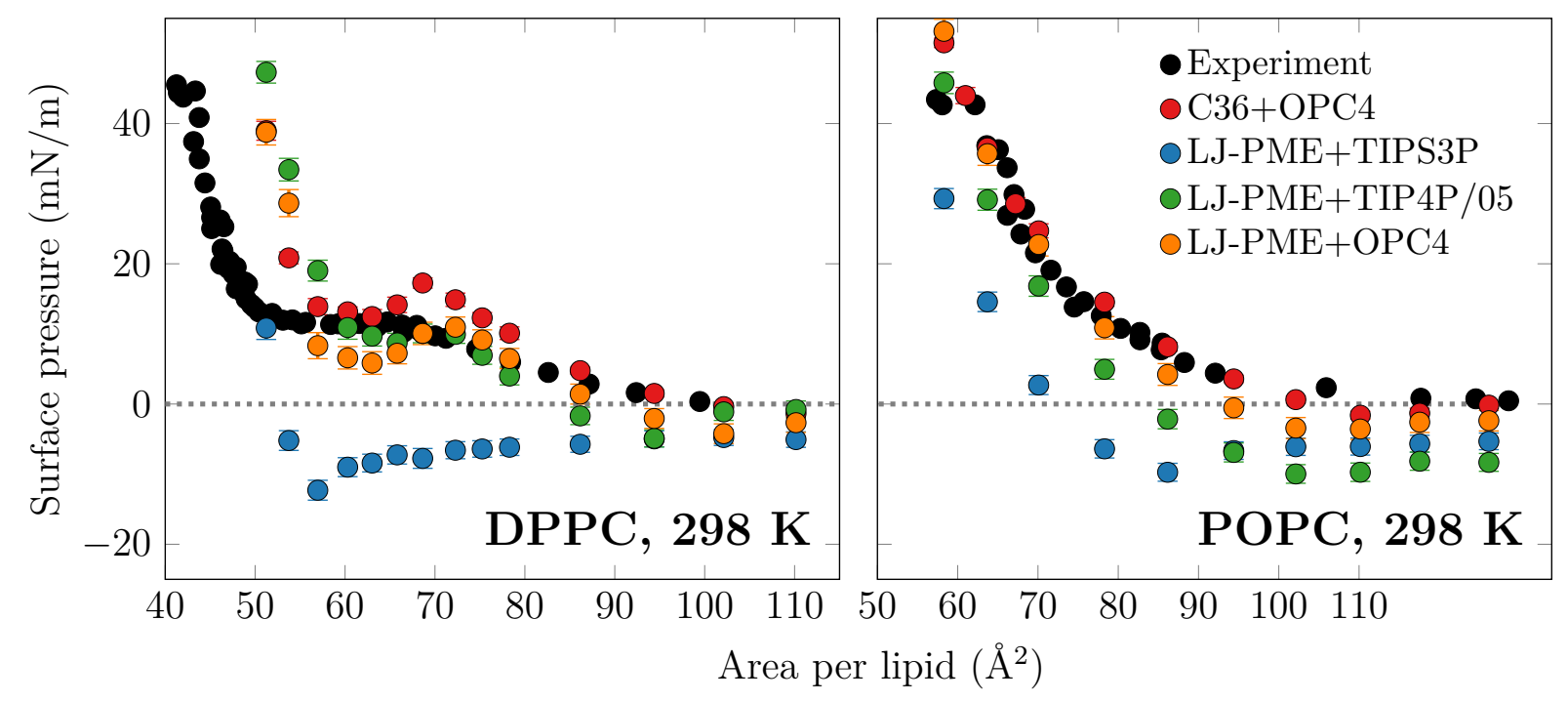

Figure 1: Surface pressure-area isotherms for DPPC and POPC at $298 \mathrm{~K}$ obtained with the C36/LJ-PME lipid model (LJ-PME) and with different water models in this work. Additionally, data for the standard C36 simulated with OPC4 water, taken from our earlier work, ${ }^{15}$ are shown together with experimental data extracted from well-equilibrated monolayers. ${ }^{49}$

To test the performace of C36/LJ-PME in monolayer simulations, we compared the surface pressure-area isotherms of DPPC and POPC monolayers with the isotherms from standard C36 with OPC4 water from our previous work ${ }^{15}$ and experiments ${ }^{49}$ in Fig. 1. Both systems are simulated at $298 \mathrm{~K}$ which is well below the $\mathrm{T}_{\mathrm{m}}$ of DPPC, yet well above 
the $\mathrm{T}_{\mathrm{m}}$ of POPC, therefore ensuring that we are not close to any phase transitions. The C36/LJ-PME with the TIPS3P water model suffers from characteristic issues for monolayer simulations performed with water models having too low surface tension: Negative surface pressures, corresponding to non-physical states where the absorbance of a surfactant layer increases the interfacial tension, appear above APL of $51 \AA^{2}$ for DPPC and $70 \AA^{2}$ for POPC in C36/LJ-PME simulations. Furthermore, stable pores appear in monolayers at an APL of $60 \AA^{2}$ for DPPC (Fig. 2) and $86 \AA^{2}$ for POPC, which are significantly below the experimental values where the gas- $\mathrm{L}_{\mathrm{e}}$ phase coexistence begins; approximately 100-110 $\AA^{2}$ and 120-130 $\AA^{2}$, respectively. ${ }^{49,50}$ The opening of pores at too small APLs can be explained by too low surface tension of the TIPS3P water model favoring the exposure of water surface rather than the transition of most lipids to the $\mathrm{L}_{\mathrm{e}}$ phase upon increasing APL. Notably, such pores may not appear in simulations with small box size due to finite size effects, ${ }^{14}$ which could be the case in monolayer simulations with 36 lipids used in the optimization protocol of C36/LJ-PME model. ${ }^{17}$

Because C36/LJ-PME with TIPS3P showed characteristic behaviour for simulations with too low water surface tension, we set out to find a water model that reproduces the experimental surface tension with LJ-PME that could be used together with the C36/LJ-PME lipid model. To this end, we evaluated the surface tension of eight water models at three different temperatures using different Lennard-Jones cutoff distances and Lennard-Jones PME in Fig. 3. Water surface tension increases in all models with the increasing cutoff, converging toward the values obtained with LJ-PME as expected. As shown also previously, OPC4 performs reasonably well with cutoffs of 1.2 and $1.4 \mathrm{~nm},{ }^{15}$ but slightly overshoots the experimental value with LJ-PME. TIP4P/05 slightly undershoots water surface tension with LJ-PME, whereas other models behave poorly, with TIP3P and TIPS3P underestimating the experimental values by $\approx 20 \mathrm{mN} / \mathrm{m}$ at all studied temperatures. Based on the results in Fig. 3, we repeated the DPPC and POPC bilayer monolayer simulations using C36/LJPME with OPC4 and TIP4P/05 water models that gave the best surface tension values with 

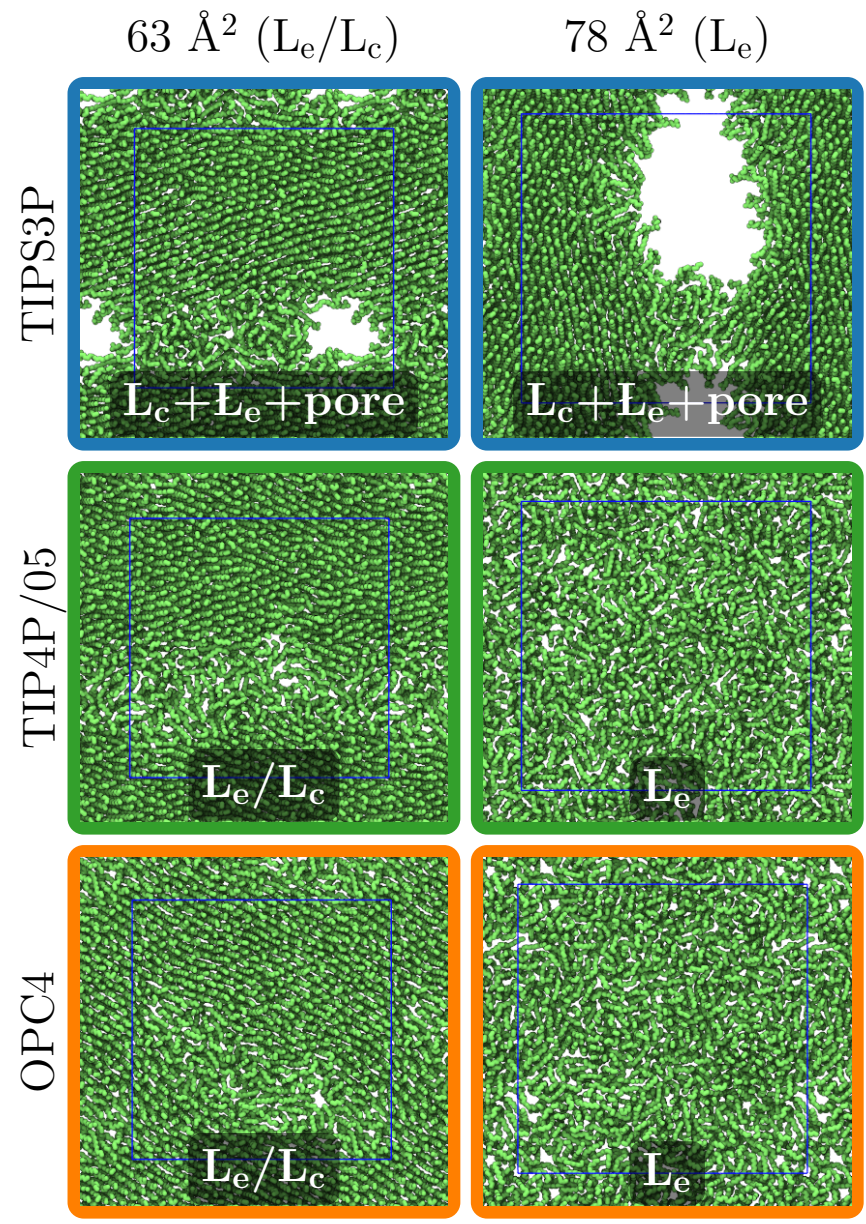

Figure 2: Snapshots of the DPPC monolayer at two APL values from C36/LJ-PME lipid model with three different water models. The labels on top indicate the expected phases based on experiments, whereas those on the snapshots show the observed ones with the used model.

\section{LJ-PME.}

Area per lipid values from DPPC and POPC bilayers simulated at different temperatures suggest that OPC4 water model is well compatible with the C36/LJ-PME parameters, yet the use of TIP4P/05 water resulted in too large APL values, especially for POPC (Fig. S1 and discussion in the SI).

Next, we calculated the surface pressure-area isotherms for DPPC and POPC with these models (Fig. 1). Large negative surface pressures were not observed in these simulations, and monolayer phase behavior was consistent with experiments and the standard C36 model 


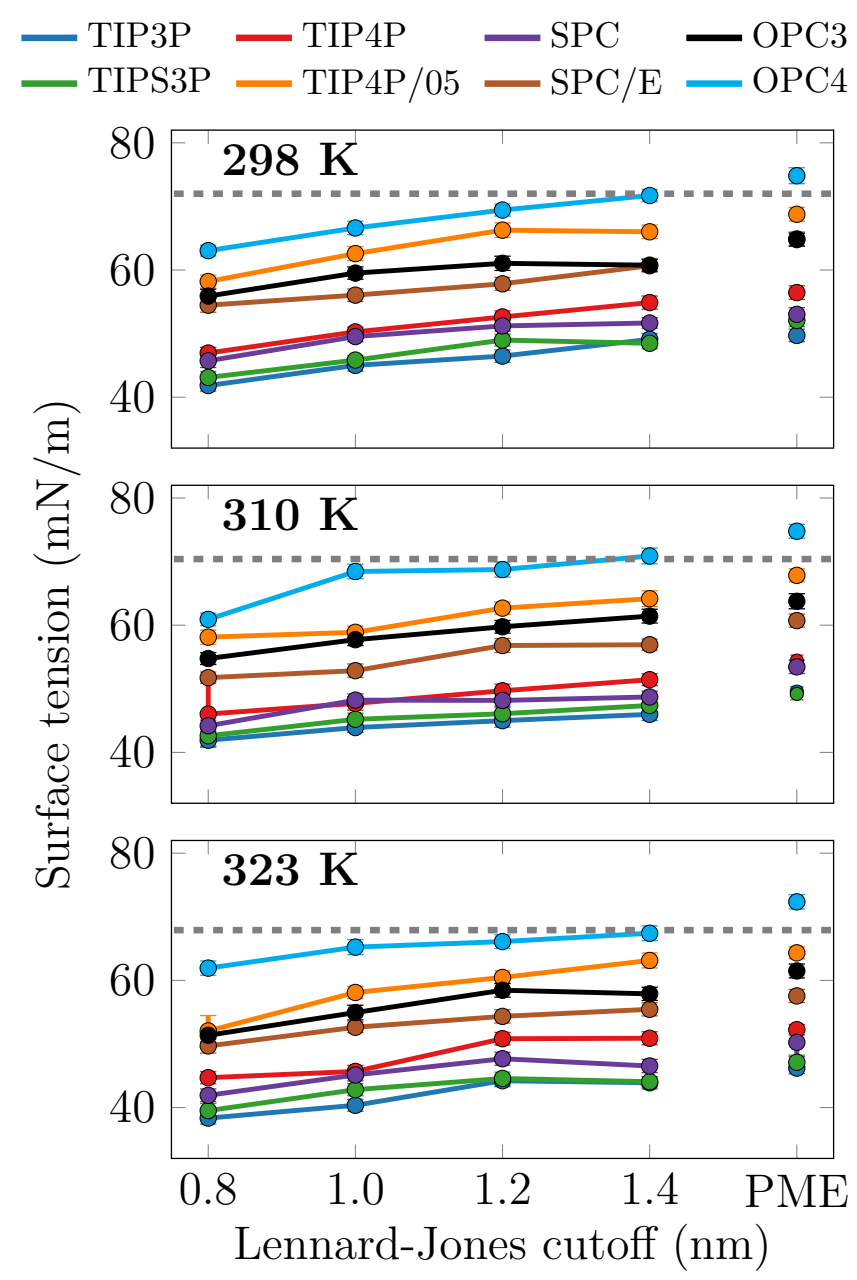

Figure 3: Surface tension of commonly used water models with different LJ cutoffs and at three temperatures.

with the OPC4 water (Fig. S3 in the SI). Instead of pore formation at too low areas, the $\mathrm{L}_{\mathrm{e}} / \mathrm{L}_{\mathrm{c}}$ coexistence was observed for DPPC, as demonstrated in Fig. 2 between areas per lipid of 57 and $75 \AA^{2}$. The surface pressure of the coexistence plateau was captured by both OPC4 and TIP4P/05. However, in the $\mathrm{L}_{\mathrm{e}}$ region of DPPC with APLs above $75 \AA^{2}$, OPC4 and TIP4P/05 undershot the isotherms from experiments and standard C36 model based on LJ cutoffs. ${ }^{15}$ For POPC monolayer, simulations with TIP4P/05 give too low surface pressure at all APLs.

Depending on the rate of compression, the experimental surface pressure-area isotherms can greatly vary in their shape and positioning. ${ }^{12}$ However, this issue is more critical for small 
APLs, whereas the behavior of more expanded monolayers is independent of the compression rate. ${ }^{51}$ Thus, we further evaluate our simulation models by analyzing the APL values where pores begin to form in DPPC and comparing these to the experimental value from vibrational spectroscopy ${ }^{50,52}$ Fig. 4 suggests approximately linear dependence between pore formation APL and surface tension of water in the simulation. However, the line fitted to the data does not pass through the experimental data point, yet an offset of $\approx 10 \mathrm{mN} / \mathrm{m}$ is observed, suggesting that also adjustments to the C36/LJ-PME lipid model are required to correctly capture the pore formation tension. In contrast, the simulations performed with the standard C36 lipid model and the OPC4 water with LJ cut-off ${ }^{15}$ are in excellent agreement with the experimental data point in Fig. 4. The discrepancy in pore forming APL may originate from the procedure to derive C36/LJ-PME parameters where parameters were fitted to reproduce the monolayer surface tension, $\gamma(\mathrm{APL})$ in Eq. (2), at three APL values. ${ }^{17}$ Because the surface tension of the TIPS3P water model, $\gamma_{0}$ in Eq. (2), is too low, parameters that reproduce the correct $\gamma(\mathrm{APL})$ lead to too low surface pressure, П(APL) in Eq. (2).

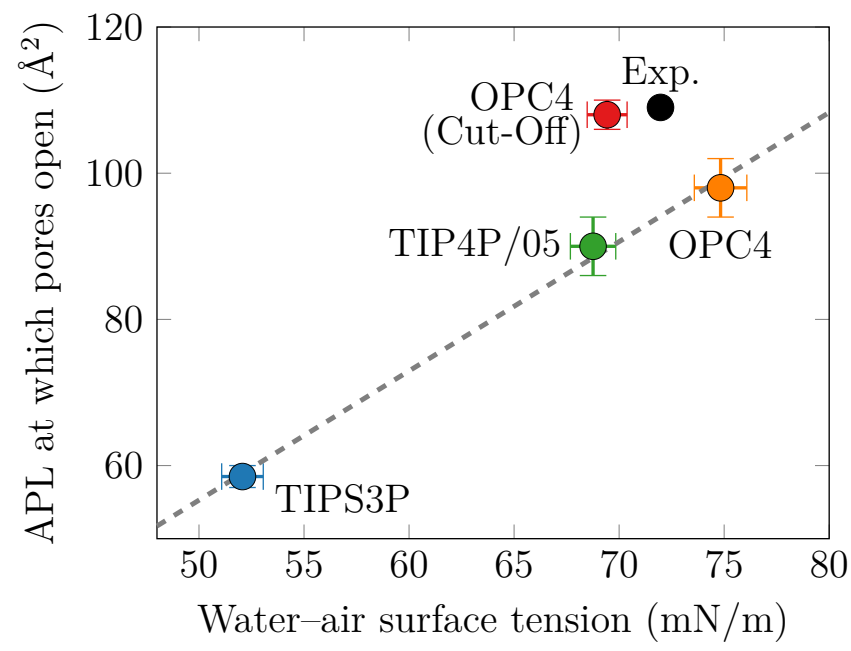

Figure 4: The dependence of the APL of pore formation in the DPPC monolayer on the surface tension of the used water model. The points simulated with C36/LJ-PME fall on a line that does not cross the experimental data point, ${ }^{50,52}$ whereas the simulation with the standard C36 lipids, OPC4 water, and LJ cut-off falls close to the experimental data point. 


\section{Conclusions}

Inclusion of long-range van der Waals interactions into C36/LJ-PME model is an important step toward more realistic MD simulations of interfaces, reducing artefacts arising, for example, from acyl chain-vacuum tension. ${ }^{16,17}$ However, C36/LJ-PME together with its standard water model, TIPS3P, fails to reproduce the experimental surface pressure-area isotherms and the expected phase behavior of DPPC and POPC monolayers. These discrepancies result from too low surface tension of the TIPS3P water model. Combining C36/LJPME lipid model with OPC4 water model - whose surface tension is closer to experimental one - considerably improves the monolayer phase behavior and agreement with experimental surface pressure-area isotherms. However, the pore forming pressure is underestimated by $10 \mathrm{mN} / \mathrm{m}$ when compared to experimental estimates.

In conclusion, the most realistic lipid monolayer simulations can be still performed by combining the OPC4 water and standard C36 lipid model with cutoff-based LJ treatment. ${ }^{15,19}$ Surprisingly, inclusion of long-range van der Waals in C36/LJ-PME did not lead to major improvements when compared with experiments, even though water model with almost correct surface tension was used.

Potential reason for this could be use of TIPS3P water model, with surface tension of approximately $20 \mathrm{mN} / \mathrm{m}$ too low, in the parametrization of C36/LJ-PME lipid model. This underestimated water surface tension is balanced by monolayer tension when optimizing against experimental surface tension values, which leads to underestimated surface pressure values. Furthermore, the small monolayers used in the optimization may get trapped into local minima with a very different surface tensions from their equilibrium values because the formation of pores in prevented by finite size effects. ${ }^{14}$

While the introduction of long-range van der Waals interactions in lipid bilayer and monolayer simulations is highly desirable, we conclude that the correct water surface tension is more critical to reproduce the experimental surface pressure-area isotherms and monolayer phase behaviour. On the other hand, increasing number of studies suggest that properties of 
water model are critical in many applications of MD simulations, such as studies of protein dynamics ${ }^{53}$ and conformational ensembles of disordered proteins. ${ }^{54,55}$ Therefore, using the state of the art water models during the systematic parametrization of force fields would most likely not only improve the description of monolayer behavior, but also facilitate other applications of MD simulations.

\section{Acknowledgement}

CT thanks the International Max Planck Research School for Many-Particle Systems in Structured Environments hosted by the Max Planck Institute for the Physics of Complex Systems, Dresden, Germany.

OHSO acknowledges the Academy of Finland (Academy Research Fellow grants 315596, 319902 and 345631) for funding.

MJ thanks the Academy of Finland (Postdoctoral Researcher grant no. 338160) and the Emil Aaltonen foundation for funding. We thank CSC-IT Center for Science (Espoo, Finland) for computational resources.

\section{Supporting Information Available}

1) Lipid bilayer simulation methods and areas per lipid of the bilayers with different water models. 2) Effect of integrator on bilayer area per lipid. 3) The phase behavior of DPPC monolayers with different water model as revealed by clustering analysis.

\section{References}

(1) Mohwald, H. Phospholipid and phospholipid-protein monolayers at the air/water interface. Annual Review of Physical Chemistry 1990, 41, 441-476. 
(2) Mouritsen, O. G. Model answers to lipid membrane questions. Cold Spring Harbor perspectives in biology 2011, 3, a004622.

(3) Blume, A.; Kerth, A. Peptide and protein binding to lipid monolayers studied by FTIRRA spectroscopy. Biochimica et Biophysica Acta (BBA) - Biomembranes 2013, 1828, 2294-2305, FTIR in membrane proteins and peptide studies.

(4) Roldán-Carmona, C.; Giner-Casares, J. J.; Pérez-Morales, M.; Martín-Romero, M. T.; Camacho, L. Revisiting the Brewster Angle Microscopy: The relevance of the polar headgroup. Advances in Colloid and Interface Science 2012, 173, 12-22.

(5) Rantamäki, A. H.; Telenius, J.; Koivuniemi, A.; Vattulainen, I.; Holopainen, J. M. Lessons from the biophysics of interfaces: lung surfactant and tear fluid. Progress in retinal and eye research 2011, 30, 204-215.

(6) Crane, J. M.; Putz, G.; Hall, S. B. Persistence of phase coexistence in disaturated phosphatidylcholine monolayers at high surface pressures. Biophysical journal 1999, r7., 3134-3143.

(7) Baoukina, S.; Tieleman, D. P. Computer simulations of lung surfactant. Biochimica et Biophysica Acta (BBA)-Biomembranes 2016, 1858, 2431-2440.

(8) Cwiklik, L. Tear film lipid layer: A molecular level view. Biochimica Et Biophysica Acta (BBA)-Biomembranes 2016, 1858, 2421-2430.

(9) Javanainen, M.; Hua, W.; Tichacek, O.; Delcroix, P.; Cwiklik, L.; Allen, H. C. Structural Effects of Cation Binding to DPPC Monolayers. Langmuir 2020, 36, 15258-15269.

(10) Rugonyi, S.; Biswas, S. C.; Hall, S. B. The biophysical function of pulmonary surfactant. Respiratory physiology \& neurobiology 2008, 163, 244-255. 
(11) Parra, E.; Pérez-Gil, J. Composition, structure and mechanical properties define performance of pulmonary surfactant membranes and films. Chemistry and physics of lipids 2015, 185, 153-175.

(12) Duncan, S. L.; Larson, R. G. Comparing experimental and simulated pressure-area isotherms for DPPC. Biophysical journal 2008, 94, 2965-2986.

(13) Klauda, J. B.; Venable, R. M.; Freites, J. A.; O'Connor, J. W.; Tobias, D. J.; Mondragon-Ramirez, C.; Vorobyov, I.; MacKerell Jr, A. D.; Pastor, R. W. Update of the CHARMM all-atom additive force field for lipids: validation on six lipid types. The journal of physical chemistry B 2010, 114, 7830-7843.

(14) Lamberg, A.; Ollila, O. S. Comment on "Structural properties of POPC monolayers under lateral compression: computer simulations analysis". Langmuir 2015, 31, 886887.

(15) Javanainen, M.; Lamberg, A.; Cwiklik, L.; Vattulainen, I.; Ollila, O. S. Atomistic model for nearly quantitative simulations of Langmuir monolayers. Langmuir 2018, 34, 25652572.

(16) Yu, Y.; Krämer, A.; Venable, R. M.; Brooks, B. R.; Klauda, J. B.; Pastor, R. W. CHARMM36 Lipid Force Field with Explicit Treatment of Long-Range Dispersion: Parametrization and Validation for Phosphatidylethanolamine, Phosphatidylglycerol, and Ether Lipids. Journal of Chemical Theory and Computation 2021, 17, 1581-1595.

(17) Yu, Y.; Kramer, A.; Venable, R. M.; Simmonett, A. C.; MacKerell Jr, A. D.; Klauda, J. B.; Pastor, R. W.; Brooks, B. R. Semi-automated optimization of the CHARMM36 lipid force field to include explicit treatment of long-range dispersion. Journal of Chemical Theory and Computation 2021, 17, 1562-1580.

(18) Izadi, S.; Anandakrishnan, R.; Onufriev, A. V. Building water models: a different approach. The journal of physical chemistry letters 2014, 5, 3863-3871. 
(19) Liekkinen, J.; de Santos Moreno, B.; Paananen, R. O.; Vattulainen, I.; Monticelli, L.; De La Serna, J. B.; Javanainen, M. Understanding the Functional Properties of Lipid Heterogeneity in Pulmonary Surfactant Monolayers at the Atomistic Level. Frontiers in cell and developmental biology 2020, 8, 581016.

(20) Jämbeck, J. P.; Lyubartsev, A. P. Derivation and systematic validation of a refined all-atom force field for phosphatidylcholine lipids. The journal of physical chemistry B 2012, 116, 3164-3179.

(21) Leonard, A. N.; Simmonett, A. C.; Pickard IV, F. C.; Huang, J.; Venable, R. M.; Klauda, J. B.; Brooks, B. R.; Pastor, R. W. Comparison of additive and polarizable models with explicit treatment of long-range Lennard-Jones interactions using alkane simulations. Journal of chemical theory and computation 2018, 14, 948-958.

(22) Wennberg, C. L.; Murtola, T.; Hess, B.; Lindahl, E. Lennard-Jones lattice summation in bilayer simulations has critical effects on surface tension and lipid properties. Journal of chemical theory and computation 2013, 9, 3527-3537.

(23) Wennberg, C. L.; Murtola, T.; Páll, S.; Abraham, M. J.; Hess, B.; Lindahl, E. Directspace corrections enable fast and accurate Lorentz-Berthelot combination rule LennardJones lattice summation. Journal of chemical theory and computation 2015, 11, 57375746.

(24) Anézo, C.; de Vries, A. H.; Höltje, H.-D.; Tieleman, D. P.; Marrink, S.-J. Methodological issues in lipid bilayer simulations. The Journal of Physical Chemistry B 2003, 10\%, 9424-9433.

(25) Huang, K.; García, A. E. Effects of truncating van der Waals interactions in lipid bilayer simulations. The Journal of chemical physics 2014, 141, 09B605_1.

(26) Jorgensen, W. L.; Chandrasekhar, J.; Madura, J. D.; Impey, R. W.; Klein, M. L. 
Comparison of simple potential functions for simulating liquid water. The Journal of chemical physics 1983, 79, 926-935.

(27) Durell, S. R.; Brooks, B. R.; Ben-Naim, A. Solvent-induced forces between two hydrophilic groups. The Journal of Physical Chemistry 1994, 98, 2198-2202.

(28) Klauda, J. B. Considerations of Recent All-Atom Lipid Force Field Development. The Journal of Physical Chemistry B 2021, 125, 5676-5682.

(29) Páll, S.; Zhmurov, A.; Bauer, P.; Abraham, M.; Lundborg, M.; Gray, A.; Hess, B.; Lindahl, E. Heterogeneous parallelization and acceleration of molecular dynamics simulations in GROMACS. The Journal of Chemical Physics 2020, 153, 134110.

(30) Vermaas, J.; Hardy, D.; Stone, J.; Tajkhorshid, E.; Kohlmeyer, A. TopoGromacs: Automated Topology Conversion from CHARMM to GROMACS within VMD. Journal of chemical information and modeling 2016, 56, 1112-1116.

(31) Eastman, P.; Swails, J.; Chodera, J. D.; McGibbon, R. T.; Zhao, Y.; Beauchamp, K. A.; Wang, L.-P.; Simmonett, A. C.; Harrigan, M. P.; Stern, C. D.; Wiewiora, R. P.; Brooks, B. R.; Pande, V. S. OpenMM 7: Rapid development of high performance algorithms for molecular dynamics. PLOS Computational Biology 2017, 13, 1-17.

(32) Izadi, S.; Onufriev, A. V. Accuracy limit of rigid 3-point water models. The Journal of chemical physics 2016, 145, 074501.

(33) Berendsen, H. J.; Postma, J. P.; van Gunsteren, W. F.; Hermans, J. Intermolecular forces; Springer, 1981; pp 331-342.

(34) Berendsen, H.; Grigera, J.; Straatsma, T. The missing term in effective pair potentials. Journal of Physical Chemistry 1987, 91, 6269-6271.

(35) Abascal, J. L.; Vega, C. A general purpose model for the condensed phases of water: TIP4P/2005. The Journal of chemical physics 2005, 123, 234505. 
(36) Páll, S.; Hess, B. A flexible algorithm for calculating pair interactions on SIMD architectures. Computer Physics Communications 2013, 184, 2641-2650.

(37) Darden, T.; York, D.; Pedersen, L. Particle mesh Ewald: An N.log (N) method for Ewald sums in large systems. The Journal of chemical physics 1993, 98, 10089-10092.

(38) Essmann, U.; Perera, L.; Berkowitz, M. L.; Darden, T.; Lee, H.; Pedersen, L. G. A smooth particle mesh Ewald method. The Journal of chemical physics 1995, 103, 85778593.

(39) Shirts, M. R.; Mobley, D. L.; Chodera, J. D.; Pande, V. S. Accurate and efficient corrections for missing dispersion interactions in molecular simulations. The journal of physical chemistry B 2007, 111, 13052-13063.

(40) Bussi, G.; Donadio, D.; Parrinello, M. Canonical sampling through velocity rescaling. The Journal of chemical physics 2007, 126, 014101.

(41) Miyamoto, S.; Kollman, P. A. Settle: An analytical version of the SHAKE and RATTLE algorithm for rigid water models. Journal of computational chemistry 1992, 13, 952962.

(42) Nosé, S. A unified formulation of the constant temperature molecular dynamics methods. The Journal of chemical physics 1984, 81, 511-519.

(43) Hoover, W. G. Canonical dynamics: Equilibrium phase-space distributions. Physical review A 1985, 31, 1695.

(44) Hess, B.; Bekker, H.; Berendsen, H. J.; Fraaije, J. G. LINCS: a linear constraint solver for molecular simulations. Journal of computational chemistry 1997, 18, 1463-1472.

(45) Hess, B. P-LINCS: A parallel linear constraint solver for molecular simulation. Journal of chemical theory and computation 2008, 4, 116-122. 
(46) Ester, M.; Kriegel, H.-P.; Sander, J.; Xu, X., et al. A density-based algorithm for discovering clusters in large spatial databases with noise. Kdd. 1996; pp 226-231.

(47) Lee, J.; Cheng, X.; Swails, J. M.; Yeom, M. S.; Eastman, P. K.; Lemkul, J. A.; Wei, S.; Buckner, J.; Jeong, J. C.; Qi, Y., et al. CHARMM-GUI input generator for NAMD, GROMACS, AMBER, OpenMM, and CHARMM/OpenMM simulations using the CHARMM36 additive force field. Journal of chemical theory and computation 2016, 12, 405-413.

(48) Pluhackova, K.; Kirsch, S. A.; Han, J.; Sun, L.; Jiang, Z.; Unruh, T.; Böckmann, R. A. A critical comparison of biomembrane force fields: structure and dynamics of model DMPC, POPC, and POPE bilayers. The Journal of Physical Chemistry B 2016, 120, $3888-3903$.

(49) Mansour, H. M.; Zografi, G. Relationships between Equilibrium Spreading Pressure and Phase Equilibria of Phospholipid Bilayers and Monolayers at the Air- Water Interface. Langmuir 2007, 23, 3809-3819.

(50) Roke, S.; Schins, J.; Müller, M.; Bonn, M. Vibrational spectroscopic investigation of the phase diagram of a biomimetic lipid monolayer. Physical review letters 2003, 90, 128101.

(51) Smith, E. C.; Crane, J. M.; Laderas, T. G.; Hall, S. B. Metastability of a supercompressed fluid monolayer. Biophysical journal 2003, 85, 3048-3057.

(52) Knecht, V.; Müller, M.; Bonn, M.; Marrink, S.-J.; Mark, A. E. Simulation studies of pore and domain formation in a phospholipid monolayer. The Journal of chemical physics 2005, 122, 024704.

(53) Ollila, O. H. S.; Heikkinen, H. A.; Iwai, H. Rotational Dynamics of Proteins from Spin Relaxation Times and Molecular Dynamics Simulations. J. Phys. Chem. B 2018, 122, 6559-6569. 
(54) Robustelli, P.; Piana, S.; Shaw, D. E. Developing a molecular dynamics force field for both folded and disordered protein states. Proc. Natl. Acad. Sci. 2018, 115, E4758E4766.

(55) Virtanen, S. I.; Kiirikki, A. M.; Mikula, K. M.; Iwaï, H.; Ollila, O. H. S. Heterogeneous dynamics in partially disordered proteins. Phys. Chem. Chem. Phys. 2020, 22, 2118521196. 


\title{
Supporting Information for:
}

\section{Accurate Simulations of Lipid Monolayers \\ Require a Water Model With Correct Surface}

\section{Tension}

\author{
Carmelo Tempra, ${ }^{\dagger}$ O.H. Samuli Ollila, ${ }^{\ddagger}$ and Matti Javanainen ${ }^{*, \downarrow, \dagger}$ \\ $\dagger$ Institute of Organic Chemistry and Biochemistry, Czech Academy of Sciences, Flemingovo \\ nám. 542/2, 16000 Prague 6, Czech Republic \\ $\ddagger$ Institute of Biotechnology, University of Helsinki, 00014 Helsinki, Finland \\ E-mail: matti.javanainen@gmail.com
}

\section{Lipid Bilayer simulations}

\section{Methods}

We simulated DPPC and POPC bilayers with the C36/LJ-PME lipid model and with three different water models and at 5 different temperatures to both validate our implementation of the C36/LJ-PME model in GROMACS, ${ }^{1}$ as well as to study the effect of different water models on the lipid bilayer behavior. DPPC and POPC bilayer simulations were set up to mimic the DPPC bilayer simulations in Refs. 2 and 3 (larger system): Bilayers with a total of 288 lipids and 31 water molecules per lipid were constructed with CHARMM-GUI. ${ }^{4}$ We performed the simulations at five temperatures for both lipid types; 323, 328, 333, 338, and $343 \mathrm{~K}$ for DPPC and 298, 303, 308, 313, and $318 \mathrm{~K}$ for POPC. In addition to the 
TIPS3P model used in the original CHARMM36/LJ-PME, ${ }^{2,3}$ we repeated the simulations with two additional water models, namely OPC4 and TIP4P/05, since they best described the surface tension of water. We performed the simulations with the simulation parameters suggested for GROMACS and CHARMM $36^{4}$ but with Lennard-Jones PME. Namely, these agree with the simulation parameters used for lipid monolayers (see main text), except that the pressures were additionally coupled semi-isotropically to a Parrinello-Rahman barostat ${ }^{5}$ with a target pressure of 1 bar, compressibility of $4.5 \times 10^{-5} 1 /$ bar, and a time constant of 5 ps. We also repeated the DPPC simulations with the standard CHARMM36 lipid model without LJ-PME, ${ }^{6}$ and for these simulations we only employed the TIPS3P water model. All simulations were $300 \mathrm{~ns}$ long, and the first $100 \mathrm{~ns}$ was omitted from analyses.

The area per lipid was extracted by calculating the total bilayer area with gmx energy, and dividing this by the number of lipids in one leaflet. The standard error was obtained from block averaging performed by gmx analyze.

\section{Evaluation of our Implementation of C36/LJ-PME in GROMACS}

Overestimated melting points for DPPC and over-condensed lipid bilayers have been reported when the standard C36 force field ${ }^{6}$ is used with GROMACS simulation engine. ${ }^{4,8}$ Therefore, we carefully evaluate here the performance of our C36/LJ-PME implementation for GROMACS comparing the DPPC and POPC bilayer APLs against previous results and experimental data in different temperatures in Fig. S1.

Our GROMACS implementation is in good agreement with the reported results from C36/LJ-PME simulations with OpenMM for DPPC at $333 \mathrm{~K}$ and for POPC at $303 \mathrm{~K} .^{2,3}$ For POPC, simulation results agree with experimental data, ${ }^{7}$ but give slightly too low values for DPPC. However, at $323 \mathrm{~K}$, the DPPC bilayer is in the ripple phase in C36/LJ-PME simulations with GROMACS, thus giving smaller APL than in experiments and OpenMM simulations. This is in line with the overestimated melting points for DPPC in the standard C36 model when simulated with GROMACS. ${ }^{8}$ 


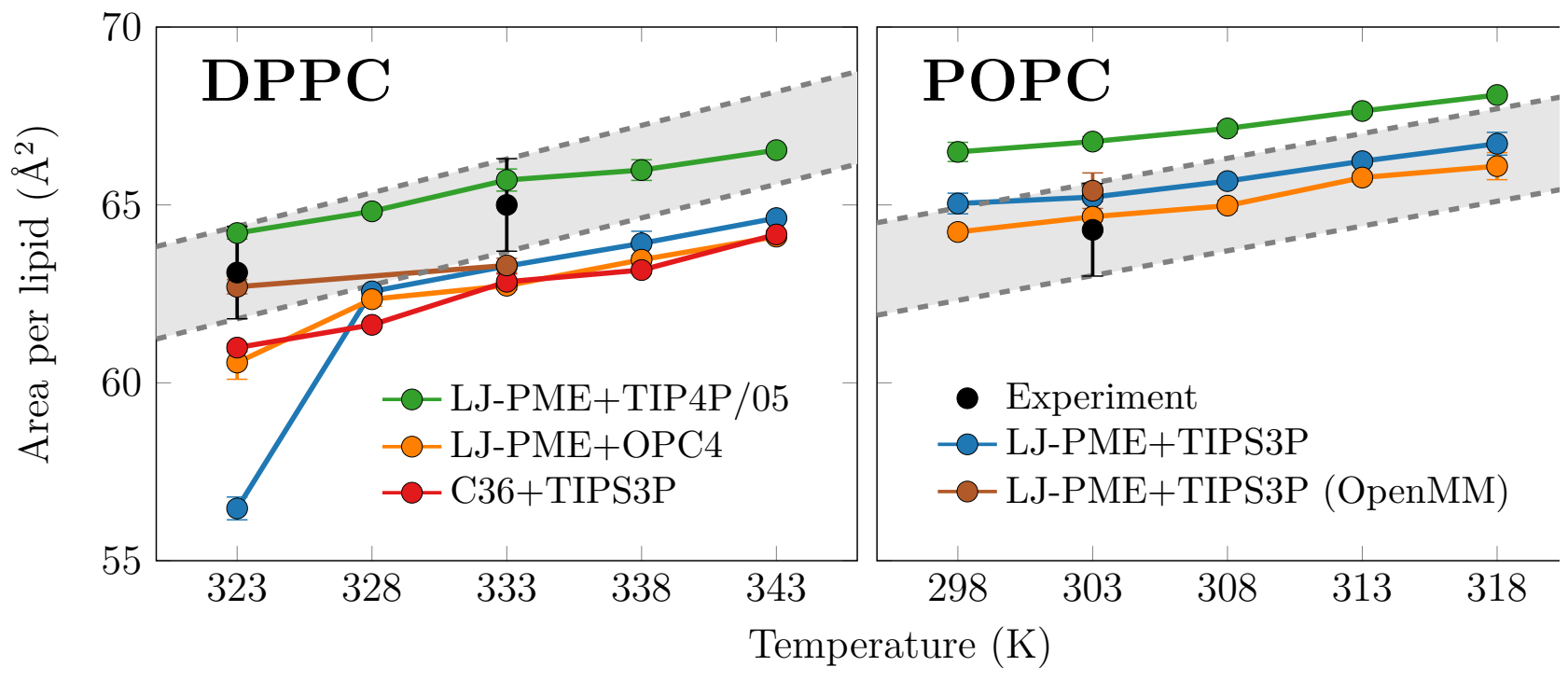

Figure S1: Area per lipid of the DPPC (left) and POPC (right) bilayers simulated at different temperatures with either the standard CHARMM36 (C36) or the C36/LJ-PME (LJPME) and with different water models. The black markers and the shaded region show the experimentally determined area per lipid and its extrapolation to other temperatures based on the thermal area expansivity values taken from Ref. 7. The data obtained with CHARMM/OpenMM in Refs. $2 \& 3$ are shown in brown.

One of the main differences between standard GROMACS and OpenMM simulation engines is the integrator; a Stochastic Langevin integrator is used in OpenMM, while a leapfrog integrator is typically used in GROMACS simulations, although the Langevin integrator is also supported. To investigate if the higher melting point in GROMACS could arise from the different integrator, we repeated the GROMACS simulation of a DPPC bilayer at $323 \mathrm{~K}$ five times using both stochastic Langevin and leap-frog integrators. These five repeats were each 1 us long. Two bottom panels of Fig. S2 demonstrate significant differences between the repeats, as expected close to phase transition. Yet, the Langevin integrator clearly provide a larger area per lipid.

Furthermore, we performed 5 repeats of the simulation with the Langevin integrator starting from a ripple-phase bilayer (the final structure of a simulation with the leap-frog integrator). The APL of these systems in the top panel of Fig. S2 demonstrates that the Langevin integrator was able to at least partially melt the ripple phase, yet the behavior of 
the five repeats was again not fully consistent.

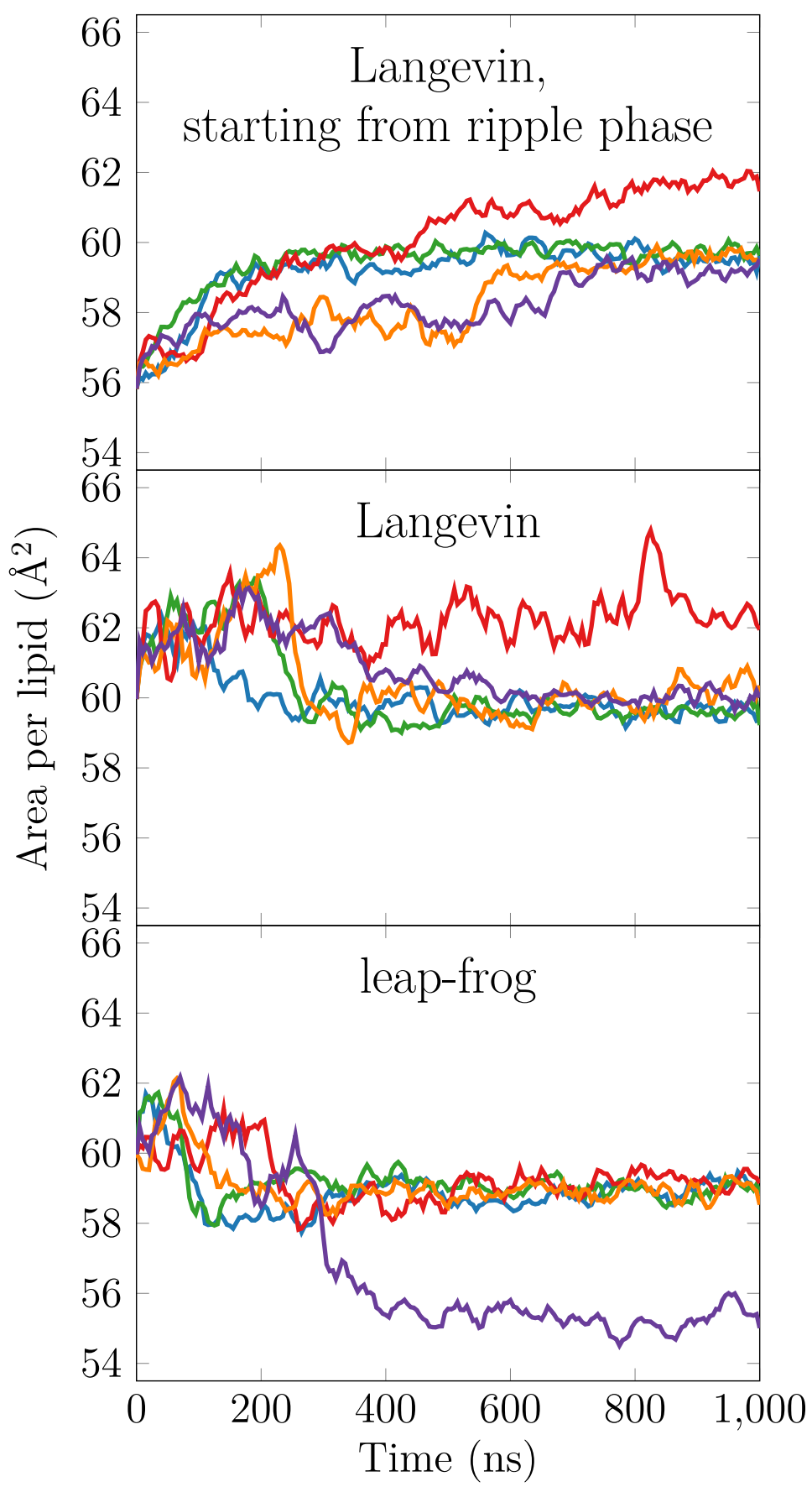

Figure S2: Time evolution of the area per lipid of DPPC at $323 \mathrm{~K}$ simulated with either the leap-frog or the stochastic Langevin integrators of GROMACS. Five repeats were performed for each simulation. The simulation corresponding to the top panel was initiated from the ripple phase, i.e. the final structure of the simulation with the leap frog algorithm. 
To conclude, our converted C36/LJ-PME parameters provide the same behavior in OpenMM, ${ }^{2,3}$ except close to the liquid-gel transition of DPPC, where small differences in the implementation of the algorithms between simulation engines and different simulation times may dominate the results.

Fortunately, interesting phenomena in DPPC monolayers studied in the main text, such as the $\mathrm{L}_{\mathrm{e}} / \mathrm{L}_{\mathrm{c}}$ phase coexistence, occur at room temperature which is well below the $\mathrm{T}_{\mathrm{m}}$ of DPPC. On the other hand, the $\mathrm{T}_{\mathrm{m}}$ of POPC falls below the freezing point of water and is thus not of importance. Therefore, the temperature in our lipid monolayer simulations is sufficiently far from $T_{m}$ of both lipids to avoid the sensitivity to simulation engine details.

Importantly, the introduction of LJ-PME does not introduce any possible new algorithmrelated sources for differences, as its implementations in these simulation engines use the same combination rule strategy, i.e. the Lorentz-Berthelot rules are used in the real space calculation, whereas the geometric ones are employed in the reciprocal space. ${ }^{9-11}$

\section{Validation of C36/LJ-PME With Alternative Water Models}

The areas per lipid of the DPPC bilayers simulated with the OPC4 and TIP4P/05 water models are shown in orange and green in Fig. S1, respectively. While OPC4 shows great agreement with experiment and slightly smaller values than the simulations with TIPS3P, the values obtained using TIP4P/05 somewhat overshoot the experimental APL values. 


\section{Lipid Monolayer Phase Behaviour}

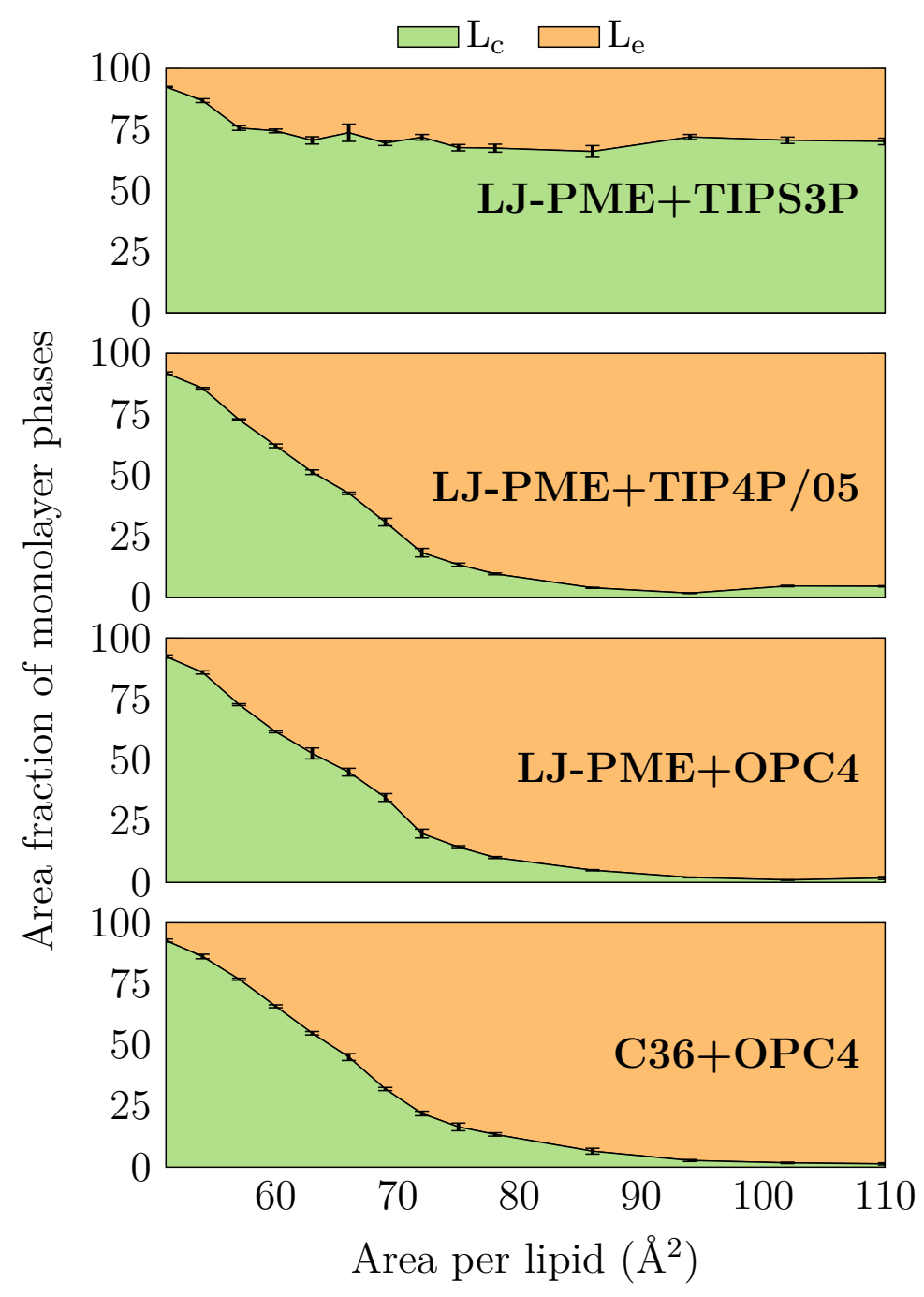

Figure S3: The fractions of chains that show $\mathrm{L}_{\mathrm{c}^{-}}$and $\mathrm{L}_{\mathrm{e}^{-}}$like packing in the DPPC monolayers. Pores are present in the monolayer with TIPS3P at areas of $60 \AA^{2}$ and larger, which results in the high $\mathrm{L}_{\mathrm{c}}$-fraction. 


\section{References}

(1) Páll, S.; Zhmurov, A.; Bauer, P.; Abraham, M.; Lundborg, M.; Gray, A.; Hess, B.; Lindahl, E. Heterogeneous parallelization and acceleration of molecular dynamics simulations in GROMACS. The Journal of Chemical Physics 2020, 153, 134110.

(2) Yu, Y.; Kramer, A.; Venable, R. M.; Simmonett, A. C.; MacKerell Jr, A. D.; Klauda, J. B.; Pastor, R. W.; Brooks, B. R. Semi-automated optimization of the CHARMM36 lipid force field to include explicit treatment of long-range dispersion. Journal of Chemical Theory and Computation 2021, 17, 1562-1580.

(3) Yu, Y.; Krämer, A.; Venable, R. M.; Brooks, B. R.; Klauda, J. B.; Pastor, R. W. CHARMM36 Lipid Force Field with Explicit Treatment of Long-Range Dispersion: Parametrization and Validation for Phosphatidylethanolamine, Phosphatidylglycerol, and Ether Lipids. Journal of Chemical Theory and Computation 2021, 17, 1581-1595.

(4) Lee, J.; Cheng, X.; Swails, J. M.; Yeom, M. S.; Eastman, P. K.; Lemkul, J. A.; Wei, S.; Buckner, J.; Jeong, J. C.; Qi, Y., et al. CHARMM-GUI input generator for NAMD, GROMACS, AMBER, OpenMM, and CHARMM/OpenMM simulations using the CHARMM36 additive force field. Journal of chemical theory and computation 2016, 12, 405-413.

(5) Parrinello, M.; Rahman, A. Polymorphic transitions in single crystals: A new molecular dynamics method. Journal of Applied physics 1981, 52, 7182-7190.

(6) Klauda, J. B.; Venable, R. M.; Freites, J. A.; O'Connor, J. W.; Tobias, D. J.; Mondragon-Ramirez, C.; Vorobyov, I.; MacKerell Jr, A. D.; Pastor, R. W. Update of the CHARMM all-atom additive force field for lipids: validation on six lipid types. The journal of physical chemistry B 2010, 114, 7830-7843.

(7) Kučerka, N.; Nieh, M.-P.; Katsaras, J. Fluid phase lipid areas and bilayer thicknesses 
of commonly used phosphatidylcholines as a function of temperature. Biochimica et Biophysica Acta (BBA)-Biomembranes 2011, 1808, 2761-2771.

(8) Pluhackova, K.; Kirsch, S. A.; Han, J.; Sun, L.; Jiang, Z.; Unruh, T.; Böckmann, R. A. A critical comparison of biomembrane force fields: structure and dynamics of model DMPC, POPC, and POPE bilayers. The Journal of Physical Chemistry B 2016, 120, 3888-3903.

(9) Wennberg, C. L.; Murtola, T.; Hess, B.; Lindahl, E. Lennard-Jones lattice summation in bilayer simulations has critical effects on surface tension and lipid properties. Journal of chemical theory and computation 2013, 9, 3527-3537.

(10) Wennberg, C. L.; Murtola, T.; Páll, S.; Abraham, M. J.; Hess, B.; Lindahl, E. Directspace corrections enable fast and accurate Lorentz-Berthelot combination rule LennardJones lattice summation. Journal of chemical theory and computation 2015, 11, 57375746.

(11) Leonard, A. N.; Simmonett, A. C.; Pickard IV, F. C.; Huang, J.; Venable, R. M.; Klauda, J. B.; Brooks, B. R.; Pastor, R. W. Comparison of additive and polarizable models with explicit treatment of long-range Lennard-Jones interactions using alkane simulations. Journal of chemical theory and computation 2018, 14, 948-958. 